\title{
Cerebral Cyst
}

National Cancer Institute

\section{Source}

National Cancer Institute. Cerebral Cyst. NCI Thesaurus. Code C3648.

A fluid filled sac within the cerebrum. It is can be congenital, trauma related or secondary to another medical condition for example an infarction. 